\title{
Italique
}

Poésie italienne de la Renaissance

VI $\mid 2003$

Varia

\section{Da Maria a Lucrezia. Su due rime giovanili di Pietro Bembo}

\section{Stefania Signorini}

\section{(2) OpenEdition}

1 Journals

\section{Edizione digitale}

URL: http://journals.openedition.org/italique/136

DOI: 10.4000/italique.136

ISSN: $1663-4438$

\section{Editore}

Librairie Droz

\section{Edizione cartacea}

Data di pubblicazione: 31 décembre 2003

Paginazione: 53-76

ISBN: 2-600-00893-4

ISSN: 1423-3983

\section{Notizia bibliografica digitale}

Stefania Signorini, «Da Maria a Lucrezia. Su due rime giovanili di Pietro Bembo », Italique [Online], VI I 2003, online dal 06 octobre 2009, consultato il 10 décembre 2020. URL : http://

journals.openedition.org/italique/136 ; DOI : https://doi.org/10.4000/italique.136

(c) Tous droits réservés 
SteFAnIA SignoRIN I

\author{
D A MARIA A LUCREZIA. \\ S U D UE RIME GIOVANILI \\ D I PIE TRO B E M B O
}




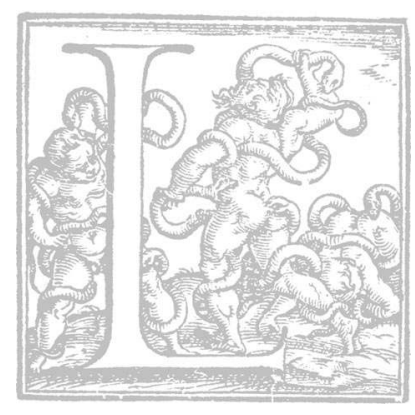

A vigilia di Natale del I502, a due mesi dall'arrivo a Ferrara, Pietro Bembo scriveva al fratello Carlo perché questi gli inviasse il manoscritto degli Asolani, che allora «circolavano [...] a Venezia per essere sottoposti al vaglio critico degli amicis. ${ }^{\mathrm{I}}$ Il trattato, in forma di dialogo, mescolando prosa e versi ed eleggendo la tematica amorosa, era in grado di reclamare all'autore l'attenzione ammirata degli ambienti cortigiani. Avviata circa il I496-97 e terminata entro l'inizio del soggiorno ferrarese, l'opera giunse alla pubblicazione solo alcuni anni più tardi, dopo un fitto lavoro revisorio che si concluse nel marzo del I505, quando Gli Asolani apparvero a stampa per i tipi di Aldo Manuzio preceduti, in alcune copie, da una lettera dedicatoria indirizzata a Lucrezia Borgia, figlia dell'ormai defunto papa Alessandro VI e sposa (in terze nozze) del duca Alfonso d'Este. ${ }^{2}$

La princeps degli Asolani, ponendosi a conclusione di un ideale segmento della vicenda artistica e biografica di Bembo (cui seguirono l'imminente soggiorno urbinate e il decisivo approdo alla corte papale), delimita una parabola ideativa ben riconoscibile, consacrata al tema amoroso e intrecciata con le personali vicende del giovane letterato veneziano. Aperta, come noto, nel nome dell'amore infelice per una misteriosa Madonna $G$ (caso specchiato nel primo libro dell'opera e parallelo alla redazione parziale della stessa, testimoniata dal codice Cl.VT.4 della veneziana Biblioteca Querini Stampalia), tale parabola prosegui nel segno della gentildonna friulana Maria Savorgnan e si concluse sotto $i$ sigilli nobilissimi della duchessa di Ferrara. Ripetuti, e in parte fruttuosi, sono stati i tentativi di ricondurre i tre libri del dialogo asolano all'esperienza privata dell'autore, specialmente assegnando a ciascun episodio biografico la tutela di una particolare concezione amorosa o ricercando nei personaggi femminili le tracce di una convergenza con le figure storiche. ${ }^{3}$ Al di là delle inevitabili contaminazioni di spunti e motivi, resta indiscutibile la simmetria con cui casi privati e ideazione artistica si avvicendarono e si affiancarono, nella biografia di Pietro, tra gli ultimi anni del '400 e il primo lustro del secolo nuovo: arte e vita, storia e poesia si incrociano a più riprese, $e$ se la produzione letteraria reca le vestigia (abilmente dissimulate) delle vicende personali, meno ovvia è la frequenza con cui l'estro poetico di Bembo occupa gli spazi della conversazione privata. É il caso - già ampiamente indagato - dei carteggi intrattenuti da Pietro con Maria e Lucrezia, dove è agevole rintracciare $i$ segni di una consapevole intenzionalità artistica, confermata dalla premura con cui uno scrittore sorvegliato, quale Bembo fu, architettò la pubblicazione (post mortem) del proprio epistolario, in aggiunta provvedendo a 
una sistemazione che, ritoccando $i$ dati storici e cronologici, si prestasse a circoscrivere un itinerario esemplare di amore e letteratura.

Maggiormente implicato con vicende spicciole e casi quotidiani, il dialogo con la Savorgnan si segnala per la vivida alternanza di preoccupazioni pratiche e passione letteraria. ${ }^{4}$ Il carteggio, che si apre con due sonetti di mano di Maria, raccoglie infatti un significativo manipolo di rime d'occasione e mescola brevi comunicazioni di servizio ad appassionate dichiarazioni d'amore nelle quali il dato storico subisce una torsione in direzione lirica ed elegiaca. Mano a mano che si avanza nella lettura della corrispondenza, la vicenda si cristallizza in una serie di emblemi e motti che restano a marcare la presenza di Maria nella produzione letteraria del Bembo.' Centrale risulta, al riguardo, la lettera 74 dove, sul limite estremo di una storia d'amore ormai vicina alla conclusione, Pietro codifica la relazione con Maria, consacrandone in via definitiva l'uscita dalla sfera del vissuto e l'ingresso in quella della letteratura:

Che voglia far di me la fortuna con tante sue offese, io non so. Bene so questo, che nè ella nè tutto il mondo potrà fare che io non v'ami, mentre io arò vita. Ahi dura, alla qual non basta così tosto avermi rotta la via, che mi portava al ben mio, facendovi lontana da me, che ancora quando doppo molti e molti pensamenti e doppo lungo tempo io alla fine mi ritrovo un dì dove voi sete, ella ogni occasione mi toglie di potere alla minor parte de' miei disiderj sodisfare, nè mi lascia pure il poter con voi un poco piagnere liberamente, non che ragionare quanto io vorrei. Sallo Idio che dolore è meco. Voglia esso che io il possa portare, che forte ne dubito, tanto nel vero mel sento tuttavia andar cingendo e abbracciando l'affannato vostro cuore, che già era mio. Volea ragionar con voi sopra quello che mi diceste, dubitando non forse sopravinto da queste ingiurie s'andasse allentando il mio amore. Oimè! che il mio amore non può per aversità di fortuna allentare, nè potrà mai. Quello che io v'ho dato di me, altro che morte non vi può torre, o di questa misera vita sostegno. Grandi sono i torti che la mia dura stella mi fa così spesso, ma molto maggiore è la fermezza del mio pensiero, il quale è, e vuole essere solo di voi sempre. Amate pur voi me, nè vi spaventino queste malagevolezze, che ci seguono amando, o vi muovano quelle cose, che muovono le altre donne tutto dì, della fortuna e volgari. Tanto più dolci saranno i frutti de' nostri amori, quanto con maggior fatiche governando le radici loro e con più nostre lagrime rigandole, gli aremo nodriti e cresciuti. Avea scritto fin qui, quando parendomi questa ultima parte acconcia materia di verso, in questi otto ne la distesi. I quali vi priego, per quanto amore mi portate, che prendiate a memoria, e cantiategli all'amico che sapete, sì quetamente che esso gl'intenda. E di questo me ne diate tosto novella, e di quanto sopra ciò fie avenuto. Ierisera la notte ci sopragiunse lontano dall'albergo con tanta fierezza di tempo tenendoci alquante ore quasi senza poter vedere dove ci andassimo, che io arei voluto più tosto essere in ogni altro luogo che in quella barca. Aspetterò intendere che arà ad esser 
di me, fatto San Giorgio. Nella qual diliberazione siavi dinanzi agli occhi questo poco contento cuor mio, che sempre in voi mirando, altro bene nè altro male vuole in questa vita che voi. Ragionate con M., ragionate con Do[nata] di me, poichè io non posso con voi ragionare. Io non tengo ora le lagrime, non tenete voi la vostra pietà.

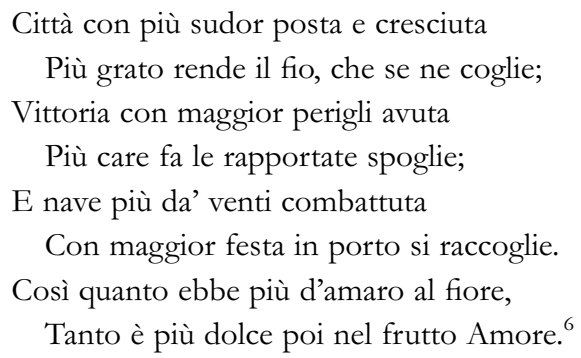

La lettera, datata dall'autore al 20 aprile I sor, risale ai mesi conclusivi della storia d'amore allorché, a seguito di un temporaneo trasferimento di Maria da Venezia a Ferrara, i convegni si erano fatti più rari e difficili. ${ }^{7}$ Ai primi di aprile, come risulta dal testo, Pietro fu in visita a Ferrara, ma - contrariamente alle attese - non riusci a incontrarsi privatamente con la donna amata. Al mancato abboccamento alludono le lamentele dell'autore, che nel finale racconta brevemente le disavventure occorsegli durante il viaggio di ritorno lungo il Po e annuncia alla Savorgnan la trepidezza con cui attende di conoscere le decisioni della donna, una volta rientrata a Venezia. Stando alle confidenze riservate alla madre, Maria aveva infatti in animo di tornare sul lido «fato San Zorzi», ovvero dopo il 23 di aprile, giorno di San Giorgio e festa patronale della città di Ferrara. ${ }^{8}$ Come andassero poi le cose non è difficile arguirlo: solo poche lettere rimangono, successive all'aprile del Iso I e l'ultima della Savorgnan, senza data ma certo non lontana dall'epilogo della vicenda, suggella - ancora una volta con un sonetto - la fine dell'idillio, che le lettere del Bembo circoscrivono al settembre dello stesso anno. ${ }^{9}$

Sullo sfondo di un equilibrio ormai fragile Pietro ordisce dunque, nella lettera del 20 aprile, una vibrante dichiarazione d'amore, in polemica resistenza alle ingiurie della sorte. Al di là dei fatti privati, la vicenda viene però raccontata attraverso il filtro di un impianto lessicale e figurativo di chiara estrazione lirica. Dopo una commossa esortazione a Maria («Amate pur voi me, nè vi spaventino queste malagevolezze, che ci seguono amando, o vi muovano quelle cose, che muovono le altre donne tutto di, della fortuna e volgari»), i segnali della letterarietà si infittiscono nella zona mediana e conclusiva del testo, dove la lettera subisce una sorta di interruzione e ripresa e la riflessione dell'autore, da individuale, si eleva a un livello sovrapersonale, approdando alla defini- 
zione di un vero e proprio assioma, destinato a divenire «acconcia materia di verso»:

Tanto più dolci saranno i frutti de' nostri amori, quanto con maggior fatiche governando le radici loro e con più nostre lagrime rigandole, gli aremo nodriti e cresciuti.

Già Dionisotti, annotando il carteggio, segnalava la coincidenza con un passo degli Asolani laddove, avviandosi a concludere la celebrazione dell'ardore sensuale, Gismondo si soffermava sulla potenza delle dolcezze amorose: «Sono care agevoli, ma disagevoli vie più care, in quanto le vittorie con alcuna fatica

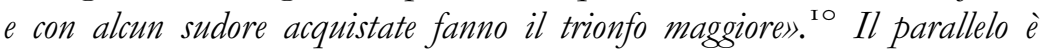
illuminante, perché conferma l'inclinazione dell'autore al travaso di temi e suggestioni dalla corrispondenza privata alla scrittura artistica e viceversa: se è difficile decidere della seriorità dell'una o dell'altra formulazione, è tuttavia doveroso ribadire la breve distanza che separa, nella produzione del primo Bembo, versante pubblico e versante privato. Il tema, del resto, doveva essere caro all'autore, che vi accenno in altri momenti del dialogo e della corrispondenza. Nel trattato, lo spunto si colora di sfumature diverse nelle parole usate da Gismondo per celebrare il futuro trionfo sul contraddittore ("Care donne, io ho sempre udito dire che vincere più gagliardo guerriere fa la vittoria maggiore») " ${ }^{\mathrm{I}}$ e nel prologo al terzo libro ("Le cose da ogniuno agevolmente possedute sono a ciascuno parimente vili, e le rare giungono vie più care»), ${ }^{\mathrm{I}}$ dove pure è riconoscibile, nell'un caso e nell'altro, la traccia mnemonica attiva nel passo sopra riferito. Nel carteggio, il concetto è anticipato in una lettera di Pietro, datata 3 I marzo I500 ma probabilmente scritta nel luglio successivo. Qui, riflettendo sui disagi affrontati dai due amanti e sulla tenacia del nodo amoroso, l'autore inserisce una tessera petrarchesca che sembra indicare un percorso privilegiato (e autorevole) nell'esegesi della vicenda sentimentale:

Increbbemi, sallo Amore, del vostro disagio. Ma che? egli non sarebbe amore altrimenti. Ora ritorno alla prima credenza: Vivace Amor, che negli affanni cresce. ${ }^{13}$

La citazione deriva dal Triumphus Cupidinis III 37, e ritorna, lievemente adattata, in una missiva della Savorgnan ascrivibile all'agosto dello stesso anno: "Non crediate che per questo mi manchi l'animo di servirvi, anzi ogni hora più si acende in amarvi perché vivace amor inegli afanni crese», dove è notevole la grazia con cui, al di là dei tratti grafico-fonetici di matrice regionale («afanni», "crese»), la Savorgnan si premurò di salvaguardare, con l'introduzione di una i- prostetica, la misura endecasillabica dell'inserto, altrimenti decurtata dall'eliminazione del relativo. ${ }^{\mathrm{I}}$ Un'ulteriore, fugace allusione al 
concetto si legge in una lettera pressoché coeva, nella quale Bembo si sofferma sui martiri inflitti a lui dalla donna e chiosa: "Ma io pensava che quello da voi fosse adoperato a posta perché il piacere, d'alcuno dispiacere attorniato mi si dimostrasse maggiore». ${ }^{\mathrm{I}}$

Il prelievo petrarchesco, denunciato nella lettera n. II e dissimulato nella n. 74 , proprio nel punto in cui più esibito è l'impianto concettuale, assicura della nobiltà del legame amoroso (elevando la relazione tra Pietro e Maria al rango dei grandi modelli classici cantati nel Triumphus) e fornisce alla teorizzazione bembesca un solido precedente. Garante Petrarca, Bembo elegge il soggetto a motivo poetico e lo traduce, congedandosi da Maria, in un breve saggio lirico, vero e proprio esercizio variantistico sul tema prescelto. Il testo è uno strambotto. La raccolta di rime di Pietro Bembo, quale fu ratificata, su volontà dell'autore, dall'edizione postuma curata da Gualteruzzi nel I548, si compone prevalentemente di sonetti e canzoni, cui si aggiunge un più ristretto manipolo di sestine, ballate e madrigali. ${ }^{16}$ Esclusi dalla silloge definitiva (come del resto dalle precedenti) rimasero gli strambotti, in numero di tre, dei quali due, poi raccolti nella sezione delle rime rifiutate, nacquero a margine della relazione sentimentale intrattenuta da Bembo con la Savorgnan e si leggono nelle pagine del carteggio pubblicato da Dionisotti. ${ }^{17}$ Il dato non è casuale e si spiega con lo statuto particolare del genere strambottistico, espressione di un fare poetico leggero e 'facile', quasi improvvisato, consono ai favori della rimeria cortese del tempo, ma lontano dal rigore petrarchesco del compiuto progetto bembiano. Di qui maturò verosimilmente l'esclusione degli strambotti dalle Rime della maturità. La scelta, mentre conferma la severità con cui l'autore costrui il proprio libro poetico, inserisce a pieno titolo il carteggio nel gusto cortigiano, in voga sul finire del XV secolo, per una lirica galante $e$ 'concettosa', graziosamente costruita mediante immagini metaforiche e probabilmente familiare alla sensibilità poetica del giovane Bembo.

Interamente articolato su una similitudine, lo strambotto posto in explicit alla lettera del 20 aprile si struttura in due sezioni, rispettivamente impegnate a descrivere il primo e il secondo membro del paragone. La prima parte occupa $i$ sei versi iniziali ed è scandita a sua volta in tre distici, ciascuno dei quali ospita un'immagine in sé compiuta: la fondazione di una città, la vittoria in battaglia, l'approdo di una nave a lungo provata dai venti contrari. La costruzione di ciascun distico è perfettamente omologa, mentre l'alternarsi di forma organica e forma non organica nei comparativi concorre a creare una struttura parallela del tipo $A A B A A B$. L'accumulo degli elementi si rivela funzionale alla preparazione del secondo e più importante momento poetico, valorizzando l'efficacia della similitudine "Cosi quanto ebbe più d'amaro al fiore, / Tanto è più dolce poi nel frutto Amore»: un amore conquistato (o salvaguardato) nonostante lunghe e sofferte traversie reca agli amanti una 
letizia maggiore. In linea con la calcolata facilità del genere prescelto, il secondo membro della similitudine è esaltato dall'adozione di un duplice stratagemma metrico-retorico. La decisione di isolare il messaggio centrale nel distico conclusivo a rima baciata crea un effetto di memorabilità che conferisce all'adagio la forza persuasiva di un sillogismo. La trouvaille si impreziosisce con l'inserzione, all'interno del paragone, di un'ulteriore figura retorica: come in un raffinato gioco a incastri, Bembo introduce una metafora nella macrosimilitudine e la relazione amorosa è presentata come percorso che procede dal corteggiamento alla gioia come dal fiore si giunge al frutto. Ė forse superfluo segnalare che lo strambotto, così costruito e chiuso da una coppia di versi che costituiscono il culmine del percorso dimostrativo, sembra ripetere, a livello strutturale e concettuale, l'immagine focalizzata dal distico finale, quasi pianta da cui fiorisce, come un bocciolo, il motto conclusivo.

Se l'impianto ideologico è petrarchesco, le modalità con cui Bembo risolse lo spunto in forma lirica non trovano nel Canzoniere un diretto precedente. Indagando tra testi ampiamente noti all' epoca, si individua dunque la sagoma di un canovaccio meno ovvio, che Bembo poteva attingere alla recente storia poetica ferrarese. Qualche decennio avanti, cantando l'amore sofferto e appassionato per Antonia Caprara, Matteo Maria Boiardo componeva la ballata che segue:

Doppo la pugna dispietata e fera

Amor m'ha dato pace,

a cui despiace che un suo servo pèra.

Come più dolce a' navicanti pare,

poi che fortuna gli ha sbatuti intorno, veder le stelle e più tranquillo il mare

e la terra vicina e il novo giorno,

cotale è dolce a me, che al porto torno

da l'unda aspra e falace,

la chiara face che mi dà lumera.

E qual al peregrin de nimbi carco

doppo notturna pioggia e fredo vento

se mostra al sole averso celeste arco,

che sol de la speranza il fa contento,

tal quel Sol ch'io credea che fusse spento

or più che mai me piace,

e più vivace è assai che già non era. ${ }^{18}$

Di là dell'innegabile differenza di ispirazione (composta e petrarcheggiante quella di Bembo, esuberante e a suo modo dantesca quella di Boiardo), la parentela tra $i$ due testi è segnalata dalla contiguità tematica e dall'omologia 
strutturale, giocata sull'adozione del paragone come elemento architettonico forte. Anche l'impianto figurativo è simile. Alla pugna boiardesca risponde la vittoria; ai navicanti (di schietta memoria purgatoriale, rafforzata dall'accostamento dell'aggettivo dolce) replica la nave. Tutta bembesca, e per ciò nuova, è invece l'invenzione conclusiva, dove l'immagine del fiore-frutto realiza uno stacco rispetto al modello e denuncia l'innesto di una tradizione ulteriore.

L'elezione della metafora vegetale acquista, in seno al carteggio con la Savorgnan, uno speciale valore emblematico. Secondo un'intuizione vulgata, suggerita da Gorni e subito accolta da Dilemmi, la radice sarebbe una delle figure adottate da Pietro per Maria. ${ }^{19}$ Accanto al motto «di pari», ${ }^{20}$ accanto alle figure della nave, del mare, del porto e della m (e del correlato valore numerico di mille), Gorni riconosce nella radice un vero e proprio senhal della giovane donna friulana, silenziosamente codificato nella corrispondenza e poi riflesso nella produzione poetica, come un filo rosso che permette (al lettore e - si può ipotizzare - alla dedicataria) di riconoscere alcune tra le liriche ispirate a Maria. La campionatura è notevole. L'emblema, mai usato dalla Savorgnan, compare per la prima volta in una lettera datata 8 luglio 1500. Subito riservata alla donna, la metafora serve a Pietro per qualificare in lei l'origine di ogni dolcezza e di ogni dolore. ${ }^{2 \mathrm{I}}$ L'allusione al nome di Maria, radice di ogni pensiero, torna in una del 22 successivo ("Nessun pensiero nasce in me da altra radice, che da quella che il vostro nome s'ba nel mio cuore barbicata») $)^{22}$ mentre, con una lieve variatio, la lettera del 9 agosto sposta metonimicamente l'emblema dalla donna all'amore: "Né crediate che questo cosi fatto pensiero sia nato da altra radice che da quella del molto e vero e incomparabile amore che io vi porto». ${ }^{23}$ L'occorrenza più interessante, avanti l'aprile I50I, si nasconde però in una pagina datata 24 luglio I 500 :

Oimè, che niun canto potrebbe ora la mia sospirosa anima dilettare, se egli già non fosse di colei, da cui ogni mio diletto vien com'ogni arbor vien da sue radici. ${ }^{24}$

Il concetto resta il medesimo. La citazione, desunta dalla canzone LXXII dei Rerum Vulgarium Fragmenta (Gentil mia donna, i’ veggio) ed esibita in clausola, suggerisce però una fonte che, con ogni probabilità, agi sulla memoria di Bembo allorché questi traghettò il senhal 'mariano' dalla prosa ai versi.

Tra le rime dedicate alla Savorgnan si individuano cinque casi di adibizione del motto, tutti puntualmente campionati nello studio di Gorni. ${ }^{25}$ La traccia petrarchesca si riverbera tuttavia in un testo ulteriore, pure ispirato all'amore per Maria, ma apparentemente estraneo al motivo arboreo: 


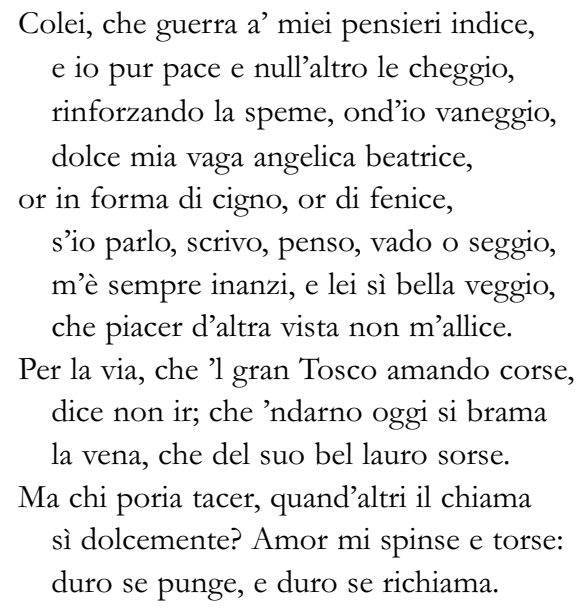

(Rime LXI)

Creando una sorte di eco in absentia, Pietro evita qui di usare il termine in questione, ma recupera la rima in -ICE, per di più adottando una delle parola-rima offerte dal modello. Beatrici (qui Beatrice, al v. 4) è infatti l'aggettivo con cui si chiude il verso petrarchesco successivo a quello citato nel carteggio e l'intero v. 4 del sonetto, derivando - in ordine - tre parole su cinque dalla fonte, ricalca il 37 della canzone: "Vaghe faville, angeliche, beatrici», con elezione della voce estrema da attributo a sostantivo. Del precedente, in aggiunta, Bembo recupera tre delle quattro rime (oltre a -ICE [-ICI in Petrarca], la rima in -EGGIO e quella in -AMA) per le quali Gentil mia donna suggerisce anche cinque delle dodici parole-rima (veggio $v . I$; seggio v. 5; beatrici v. 37; brama v. 68; chiama v. 73). E probabile che, dialogando a distanza di questioni poetiche e del valore modellizzante della poesia del "gran Tosco», Bembo volesse qui segnalare al lettore colto una chiara trama petrarchesca, contraddicendo nei fatti, prima che nelle parole, alle proposte eversive della Savorgnan, che lo invitava a ricusare il modello lirico proposto dal Canzoniere.

La presenza di Maria dietro la lirica LXI è stata ampiamente provata da Gorni, al quale si deve anche la segnalazione della comune origine che lega il già citato v. 4 del sonetto ("dolce mia vaga angelica beatrice») al v. II di una canzone (pure 'mariana': A quai sembianze) inserita nella prima veste del libro asolano ( 1505 ) e poi espunta dall' autore in prossimità della ne varietur ("la dolce vista angelica beatrice»). ${ }^{26}$ La parentela tra $i$ due passi, usciti, su memoria petrarchesca, «dallo stesso stampo», ${ }^{27}$ rinforza l'idea che la rima in -ICE sia stata inserita in LXI proprio al fine di creare una sorta di eco in grado di evocare l'emblema della radice (parola-rima della canzone A quai sembianze) e, dunque, di riflettere l'immagine di Maria Savorgnan. La 
struttura rimica del componimento preso in esame consente anzi di suggerire un'ipotesi ulteriore, segnalando un sonetto forse ascrivibile al nome della gentildonna friulana:

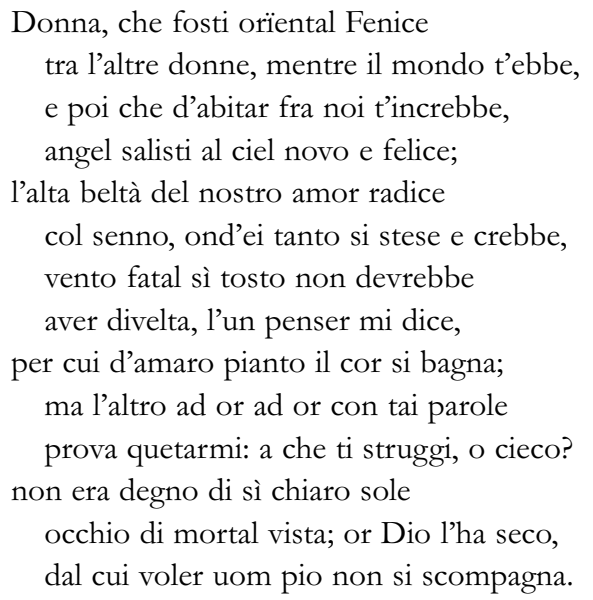

(Rime CLV)

Inserito nella sezione in morte e dedicato al compianto di una giovane donna, il sonetto CLV delle Rime di Bembo viene tradizionalmente assegnato all'amore per Faustina Morosina della Torre. In mancanza di una datazione certa e di prove inconfutabili, alcune spie generano tuttavia più di un dubbio circa la giustezza dell'attribuzione. L'uso della metafora al v. 5, qui tradotta nell'idea della bellezza come fonte prima dell'amore, indica una possibile pista 'mariana', avallata dall'ipotesi di Gorni che altri tre sonetti in morte debbano la loro ispirazione alla memoria della Savorgnan. ${ }^{28}$ La rima di radice con felice $e$ Fenice, inoltre, collega il sonetto alle restanti liriche per Maria: se infatti il primo termine (felice) ricorre con frequenza nei componimenti in cui compare l'emblema arboreo, l'immagine mitica della Fenice circoscrive un tópos che conta, nel canzoniere, due sole occorrenze, la prima delle quali è contenuta proprio nel sonetto LXI. ${ }^{29}$ La trasfigurazione della donna, infine, salita al cielo in forma di angel ( $v .4)$, mentre recupera un'evidente soluzione stilnovistica, potrebbe evocare l'«angelica beatrice» dello stesso sonetto LXI, al quale dunque la lirica in questione risulterebbe legata a filo doppio.

Quali furono le ragioni che indussero Pietro a scegliere proprio il motivo della radice come cifra della donna amata è questione che si può tentare di risolvere pensando ai motti del porto del mare e della nave, già assegnati dall'autore alla Savorgnan. Seguendo la strada della tradizione liturgica, è possibile immaginare l'eco scritturale implicita nelle immagini citate, laddove queste 
venissero riservate a una donna di nome Maria. Tali emblemi riconducono infatti al sistema simbolico adibito alla Vergine e codificatosi, nel corso dei secoli, su base biblica e patristica. La simbologia mariana, formalizzata nella sua versione più celebre dalle litanie lauretane, aveva raggiunto alla fine del $X V$ secolo una solidità e una diffusione tali da rendere verisimile la sua adozione da parte del giovane Bembo. ${ }^{30}$ Una possibile sorgente mariana potrebbe avere anche l'allusione, in seno al carteggio, al tema delle rose, forse non dimentico dell'immagine tràdita della Madonna come rosa mystica. Presente sia nelle lettere della Savorgnan che in quelle del Bembo, l'immagine sembra avere un valore emblematico nella conversazione tra $i$ due amanti, che in un caso (Savorgnan s6 e Bembo I2, risposta alla precedente) indugiano su un accenno alla «stagione lunga delle rose». ${ }^{3 \mathrm{I}}$ Replicando con la radice lo schema ideologico proposto, in virtù della coincidenza onomastica Bembo può adattare alla Savorgnan un senhal che la tradizione scritturale e innologica voleva consacrato alla Vergine e del quale quella medesima tradizione diventa garanzia e chiave esegetica. La fonte, trasparente, è un passo del libro di Isaia: "Et egredietur virga de radice Iesse, et flos de radice ejus ascendet» (Is. II, I). Dietro l'apparente facilità, il criterio con cui Bembo procede alla ratifica è però piuttosto raffinato: allusa in via implicita nelle lettere precedenti e nella produzione lirica, la fonte veterotestamentaria subisce una sorta di disvelamento proprio nella lettera del 20 aprile, non a caso occorrenza estrema del motto arboreo all'interno del carteggio. Riflettendo su una vicenda amorosa ormai prossima ad esaurirsi, Bembo rinnova la metafora vegetale, questa volta adattandola a un più vasto ragionamento de amore. Nell'atto di tradurla in poesia, inoltre, egli recupera, variandole, le tessere scritturali, evitando l'allusione diretta alla radice (che si affaccia tuttavia dalla sezione in prosa), ma replicando l'impianto concettuale del passo biblico nell'idea del frutto profano (l'amore) che sboccia dal fiore (la sofferenza), come il fiore sacro (Cristo, l'Amore divino) era germogliato dalla radice di Iesse (la Vergine, alla quale, come noto, Simeone aveva predetto: "Et tuam ipsius animam pertransiet gladius» Lc 2, 35).

La mescolanza di prosa e versi, garantendo un'elegante via di corteggiamento, offriva a Pietro uno strumento in grado di coniugare vicenda privata e vocazione artistica. Le liriche che accompagnano le lettere a Maria, oltre ad assolvere un'innegabile funzione galante, denunciano la volontà, poetica e letteraria, di tradurre in versi le idee espresse nella prosa. Il modello lontano restava, ovviamente, la Vita Nova, ma non si può trascurare l'incidenza della coeva, parallela esperienza degli Asolani, dove Pietro andava sperimentando, questa volta in maniera sistematica, una scrittura ragionativa, dimostrativa e lirica al tempo stesso, in aggiunta discutendo gli stessi temi che trovavano spazio tra le righe della corrispondenza privata. 
Non è un caso che l'identico motivo assunto a soggetto dello strambotto per Maria venga replicato, con un evidente processo dilatatorio, in una lettera più tarda, indirizzata a una certa Mad. N. (Madonna Nicola, "donzella della Duchessa di Ferrara», ${ }^{32}$ come si evince da una comunicazione successiva), ma verosimilmente destinata a colei che, per contingenza storica e per elezione, era diventata l'ultima musa degli Asolani. ${ }^{33}$ Scrivendo a Lucrezia Borgia, Pietro ribadisce la fedeltà del proprio amore di fronte alle avversità della sorte. Si ripete qui il passaggio fondamentale:

E allora ci fia caro e dolce ricordarci d'esser fermi e constanti amanti stati, e parracci d'essere pur solo per tal memoria felici: con ciò sia cosa che le vittorie più sudate e più faticate fanno il trionfo maggiore e più caro. ${ }^{34}$

L'asserto ricalca fedelmente una sentenza di Gismondo e si inserisce in un contesto in cui vengono riutilizate numerose tessere lessicali desunte dagli ultimi capitoli del secondo libro asolano. ${ }^{35}$ La contiguità riesce ancor più suggestiva se si accoglie la datazione proposta da Bernardo Morsolin che, fissando la lettera al febbraio Isos (un mese avanti la princeps degli Asolani), la pone implicitamente a reagire con il testo del dialogo, all'epoca oggetto delle cure estreme dell'autore. In ogni caso il passo segnalato suggerisce anche per il carteggio con la duchessa di Ferrara (come già per quello con Maria) l'ipotesi di una forte intenzione letteraria, accentuata semmai, nel caso di Lucrezia, dalla speciale posizione della donna amata, rispetto alla quale Bembo si poneva nell' atteggiamento rispettoso e guardingo del suddito fedele. La corrispondenza tra Pietro e Lucrezia si avviò ai primi di gingno del I503 e prosegui, prima fitta, poi (dopo la separazione) sempre più rarefatta, fino all'autunno del I5I7. Del carteggio, al di là dei dati biografici, interessa lo speciale trattamento riservato da Bembo al tema amoroso. Il quale, intrecciato con preziosi omaggi cortigiani, manifesta una natura lirica indubbiamente più spiccata di quanto non accadesse nelle lettere per Maria. L'amore fa la sua comparsa nelle pagine dell'epistolario con la lettera dell'8 giugno I 503 allorché, assecondando una richiesta della duchessa, Bembo suggeriva a Lucrezia una massima da apporre su una medaglia raffigurante una fiamma: «Al fuoco, nell'oro che V.S. m'ha mandato da farvi alcun motto sopra, da portar per impresa, non ho saputo dar miglior luogo che l'anima. Perciò potrete inscriverlo cosi: Est animum». ${ }^{36}$ La fiamma era destinata a divenire uno degli emblemi dell'amore di Pietro per la Borgia ${ }^{37}$ e la successiva del Bembo, dissimulata dietro il pretesto dei ragionamenti di corte, formula la prima compiuta dichiarazione d'amore:

Poi ch'ogni ardir mi circonscrisse Amore

Quel dì ch'io posi nel suo campo il piede, 
Tanto ch'altrui non pur chieder mercede,

Ma scoprir sol non oso il mio dolore,

Avess'io almen d'un bel cristallo il core,

Che quel ch'io taccio e Madonna non vede

Dell'interno mio mal, senza altra fede,

A' suoi begli occhi tralucesse fore;

Ch'io crederei della pietate ancora

Veder tinta la neve di quel volto,

Che 'l mio sì spesso bagna e discolora.

Or che questo non ho, quello m'è tolto,

Temo non voglia il mio Signor ch'io mora,

Ché la difesa è poca, e 'l strazio è molto.

Mirando questi dì nel mio cristallo - del quale si ragionò l'ultima sera che io a V.S. feci riverenza - ho nel mezzo di lui letti questi versi che fuori mi traluceano; i quali ora scritti vi mando in questo foglio. Dolcissimo mi sarebbe, e sopra ogni tesoro caro, che V.S. a me facesse vedere allo 'ncontro alcuna cosa che essa avesse letta nel suo. Il che tuttavia non so bene se io mi debba sperare che facciate, considerando che voi l'altro ieri ancora di quelle cose mi taceste, che proposto m'avevate di ragionarmi. Bascio a V.S. la mano. ${ }^{38}$

Era il I9 giugno I503. Con l'allusione alle conversazioni intrattenute "l'ultima sera che io a V.S. feci riverenza», Pietro associava il ricordo di un probabile, discreto corteggiamento con l'evocazione di una pratica certamente diffusa, più tardi messa a frutto nel Libro del Cortegiano, che eleggeva la corte a luogo di nobili discussioni teoriche sui temi della cultura, dell' arte, della vita di società e persino dell'amore. Un esempio vicino, nel tempo e nello spazio, era proprio il dialogo asolano, soluzione 'cortese' e punta di un iceberg che comprendeva una ricca messe di trattati de amore. Il rinvio al recente colloquio serale giustifica la particolare struttura dell'epistola che, anziché presentare un concetto in forma discorsiva per poi restituirne la trascrizione lirica (come accadeva nella lettera indirizzata alla Savorgnan), si apre immediatamente con un testo poetico, offerto all'amata come traduzione ed epilogo del comune conversare. La replica, datata al 24 giugno seguente, costituisce la ratifica del legame tra le parti, tanto più autorevole in quanto concessa da colei che sola poteva, per genere e per nobiltà, ammettere l'amante al ruolo di pari:

Miser Pietro mio, circha el desiderio tenite intender da me lo incontro del vostro o nostro cristallo, che cusì meritamente se po reputar e chiamare, non saperìa mai che altro posserne dire o trovarçe salvo una estrema conformità, forsi mai per nisun tempo igualata. E questo basti, e risti per evangelio perpetuo.

Questo da qui avante serrà el mio nome: FF. 
La lettera inaugurava, in explicit, quello che sarebbe diventato il senhal amoroso di Lucrezia, (poi per lungo tempo celata, nella corrispondenza, dietro una sigla che attende ancora una parafrasi definitiva) ${ }^{39}$ e ammetteva l'immagine del cristallo a emblema ulteriore della vicenda amorosa, assecondando la fantasia poetica messa in campo da Pietro nel sonetto, poi VII della raccolta di Rime. $^{40}$

La lirica ebbe notevole fortuna e si rivela sapientemente architettata, dotata di una forza poetica che, giustificando l'ammissione al canzoniere, ne dichiara la superiorità rispetto al pur godibile esito strambottistico sopra analizzato. Il testo ha struttura circolare, divisa in due parti dal punto fermo del v. II che, isolando gli ultimi versi, conclude l'ampio giro sintattico che coinvolge le due quartine e la prima terzina. Tra i primi otto versi e $i$ successivi, inoltre, si registra una sorta di continuità fonetica, creata attraverso la consonanza ricca che lega la rima in -ORE alla rima in -ORA. Il poeta muove da un dato reale, dichiarandosi captus Amore, e introduce il tema della passione come forza paralizzante che soggioga l'innamorato. Segue, nella seconda quartina e nella prima terzina, il passaggio a una dimensione ipotetica, giocata sull'immagine del cuore-cristallo, inteso come espediente in grado di ottenere la pietà della donna, apertamente mostrando le sofferenze patite dal poeta. L'epilogo, negando la dimensione desiderativa, chiude il cerchio e recupera la situazione reale: il cuore non è di cristallo e sull'innamorato, privato dell'amore corresponsivo della donna (giusta il magistero dantesco), incombe la minaccia della morte. Il sonetto, concettosa variatio sul tema del cuore dell'innamorato, svolge, ampliandolo ed eleggendolo a motivo unico del componimento, uno spunto nascosto tra le righe della canzone petrarchesca Sì è debile il filo a cui s'attene:

\begin{abstract}
Certo, cristallo o vetro non mostrò mai di fore nascosto altro colore, che l'alma sconsolata assai non mostri piú chiari i pensier nostri, et la fera dolcezza ch'è nel core, per gli occhi che, di sempre pianger vaghi, cercan dì et nocte pur ch'i' glien'apaghi
\end{abstract}

(Rvf. 37, 57-64)

Il precedente sembra messo a frutto già in un passo di una canzone asolana, dove tuttavia al vetro si sostituisce uno specchio: «E sì come di speglio / un riposto colore / saglie talor e luce in altra parte, / cosi di queste carte / rilucesse ad altrui / la mia celata gioia». ${ }^{41}$ Più complessa è la soluzione 
affidata al sonetto per la Borgia, nel quale il recupero della preziosa metafora cristallina si presta a una più vasta diversione sul tema.

Oltre lo spunto iniziale, il debito contratto con il Canzoniere si dichiara specialmente all'analisi linguistica. Se dantesco è il circonscrisse del v.I (sconosciuto ai Rvf.), schiettamente petrarchesca è infatti la restante trama lessicale: oltre ad ardir, mercede, dolore, core, begli occhi, pietate, $e$ volto, è soprattutto tralucere ad accusare la memoria del 'campione' di Laura. In seno ai Rerum Vulgarium Fragmenta il verbo ba 7 occorrenze, invariabilmente associate alle immagini del cuore e del vetro:

dentro là dove sol con Amor seggio, quasi visibilmente il cor traluce.

$$
(R v f .72,5-6)
$$

Poi che vostro vedere in me risplende, come raggio di sol traluce in vetro

$$
\text { (Rvf. 95, 9-10) }
$$

Ma freddo foco et paventosa speme de l'alma, che traluce come un vetro,

$$
\text { (Rvf. I 47, IO-I I) }
$$

Imaginata guida la conduce, ché la vera è sotterra, anzi è nel cielo, onde piú che mai chiara al cor traluce

$$
\text { (Rvf. 277, 9-I I) }
$$

Già traluceva a' begli occhi il mio core

$$
\text { (Rvf. 317, 5) }
$$

D'un bel diamante quadro, et mai non scemo, vi si vedea, nel mezzo, un seggio altero ove, sola, sedea, la bella Donna; dinanzi, una colonna cristallina, et iv'entro ogni pensero scritto, et for tralucea sí chiaramente che mi fea lieto, et sospirar sovente.

$$
\text { (Rvf. 325, 24-30) }
$$

[...] et tanta luce dentro al mio core infin dal ciel traluce (Rvf. 357, 6-7) 
La prima segnalazione prodotta (e il rilievo non è forse privo di significato) riconduce alla canzone Gentil mia donna, i’ veggio, che si è vista già attiva nella memoria poetica di Pietro. Interessante è anche il caso di Rvf. 325, penultimo della campionatura, dove il verbo tralucere, associato all'idea del diamante, è messo esplicitamente a contatto con l'aggettivo cristallina, quasi suggerendo la soluzione poi attuata dall'epigono. Del resto, a conferma della fedeltà al modello, ritoccando le rime in vista della stampa Bembo intervenne sul verso finale del sonetto, sostituendo «strazio» con «languir», verosimilmente più rispettoso dell'omogeneità linguistica (in senso elegiaco) codificata dal Canzoniere. ${ }^{42}$

Dunque nelle rime per Lucrezia, su autorità petrarchesca, lucentezza, trasparenza e visibilità (unite all'idea implicita di una tempra - cristallina estranea al poeta ma forse ascrivibile alla donna amata) si definiscono come vettori privilegiati del rapporto amoroso. Il sonetto del gingno I503 pare anzi proporsi come intenzionale sigillo del sogno lucreziano, collettore dei caratteri attorno a cui si focalizza l'immagine poetica della Borgia. Si tratta di un universo simbolico riducibile al sema ultimativo della luce, estrosamente associato, secondo una fantasiosa etimologia segnalata da Gorni, al nome dell' amata. ${ }^{43}$ Accanto al fuoco, alla luce $e$ al sole (con il tema correlato del vedere), il cristallo definisce un'area concettuale affine, condividendo con le precedenti alcuni tratti fondamentali: preziosità, luminosità, limpidezza, caldo/freddo (per via di contrasto) e purezza. Tale sistema si riverbera, franto, replicato e dissimulato, nella zona centrale del testo: alla semantica del vedere conducono scoprir, vede, occhi, tralucesse $e$ veder; alla luce rinviano cristallo, begli occhi $e$ tralucesse; al freddo e al caldo (e ai corrispondenti valori coloristici di bianco e rosso) alludono cristallo, pietate, tinta, neve $e$ discolora. Il mito di Lucrezia si stava plasmando.

"Con lettera del is dicembre Iso6 da Urbino il Bembo inviava a Lucrezia Borgia "due sonetti nati di poco sopra la impresa del reverendissimo cardinal di Aragona: E per più non poter fo quant'io posso"》». ${ }^{44}$ Della coppia, XLII e XLIII delle Rime, interessa qui il primo elemento, per il quale è lecito ipotizzare una consapevole derivazione dal sonetto VII:

Se dal più scaltro accorger de le genti portar celato l'amoroso ardore in parte non rileva il tristo core né scema un sol di mille miei tormenti, sapess'io almen con sì pietosi accenti quel, che dentro si chiude, aprir di fore, ch'un dì vedessi in voi novo colore coprir le guancie al suon de' miei lamenti.

Ma sì m’abbaglia il vostro altero lume, 
ch'inanzi a voi non so formar parola e sto qual uom di spirto ignudo e casso.

Parlo poi meco e grido e largo fiume verso per gli occhi, in qualche parte sola, e dolor, che devria romper un sasso.

(Rime XLII)

A tre anni di distanza dalla lettera che aveva inaugurato il gioco amoroso con Lucrezia, lasciata ormai Ferrara e raggiunta la corte feltresca di Urbino, Pietro volle con ogni probabilità rinverdire nella duchessa il ricordo delle prime schermaglie. E lo fece tramite l'invio di una lirica che, replicando nella struttura e nell'impianto figurativo il sonetto antico (si notino il calco puntuale del v. , e la perfetta corrispondenza tra le due quartine), trasferiva la riflessione dal piano del vedere a quello del dire, più congeniale alla sopravvenuta separazione dei due amanti, ai quali la distanza precludeva per il momento una possibilità di incontro. Alla vista si sostituisce dunque la parola, nuovo tramite (ancora una volta delusorio) del disvelamento amoroso. Ma la conclusione del sonetto, con l'immagine già petrarchesca dell'innamorato infelice, abbandonato alle lacrime e ridotto a una disperata solitudine (su memoria dell'Orfeo virgiliano), sancisce nuovamente una condizione di scacco, ultimamente ribadita dall'epilogo del sonetto seguente, dove si legge la ratifica definitiva dell'amore 'impossibile' per Lucrezia Borgia: E per più non poter fo quant'io posso.

Quanto egli poteva era, ancora una volta, tradurre l'amore in letteratura. Proseguendo su una strada iniziata anni prima, ai tempi della passione per Madonna G. e del primo abbozzo degli Asolani, Pietro sceglieva nuovamente di spendere la vicenda biografica in direzione artistica. La contiguità tra le due sfere (privata e poetica) affiora già alla lettura del giovanile dialogo, rispetto al quale la corrispondenza amorosa riveste il ruolo di informale controcanto. Dietro e oltre le intenzioni cortigiane emerge, nei carteggi con Maria e Lucrezia, la volontà di elaborare una precisa 'teoria dell'amore', di cui le lettere costituiscono tasselli che vanno ad aggiungersi ai pensieri espressi nel prosimetrum degli Asolani, integrandoli o traducendoli in forma confidenziale $e$ intimistica. $^{45}$

Confluita nel crogiolo ideativo degli Asolani, l'esperienza privata trovò ulteriore soluzione artistica nella raccolta delle Rime. Selezionando i prodotti poetici in vista della ne varietur, Bembo provvide a creare un sistema coeso, dove le tracce delle muse (da Maria alla Morosina) si incrociano e si sovrappongono, ora confondendosi, ora lasciandosi riconoscere, in un equilibrio tensivo che sostituisce, all'univocità della lirica petrarchesca, la mobile plurivocità del nuovo canzoniere. Con l'eccezione di qualche illustre esclusa, le rime un 
tempo destinate (e talora spedite) alle donne amate si redistribuiscono nella silloge, magari adunandosi per drappelli che formano minuscole serie implicitamente intitolate a una medesima dama. ${ }^{46}$ A risolvere la spesso intricata questione delle attriburioni dei componimenti all'una o all'altra restano, a tratti decisivi, $i$ segnali custoditi dalla corrispondenza privata, vero e proprio codice di accesso (in parte ancora da decifrare) al sistema emblematico costruito da Bembo per le sue donne.

Stefania Signorini 
I. C. Berra, La scrittura degli "Asolani” di Pietro Bembo, Firenze, La Nuova Italia, I995, p. 173.

2. Sulla vicenda redazionale degli Asolani, oltre al saggio di Claudia Berra, è imprescindibile: Pietro Bembo, Gli Asolani, edizione critica a c. di G. Dilemmi, Firenze, Accademia della Crusca, I99 (Scrittori italiani e testi antichi pubblicati dall'Accademia della Crusca, I 2). La dedica a Lucrezia si legge solo in alcune copie della princeps, verosimilmente - come segnala Clough - a causa della fretta con cui la stampa venne allestita e in attesa di ottenere dalla duchessa il consenso definitivo alla pubblicazione di quella che, in origine, era una lettera privata. Si vedano C. H. Clough, Pietro Bembo's "Gli Asolani” of I505, «Modern Language Notes», LXXXIV (1969), I, pp. I6-45 e, dello stesso, The printing of the first edition of Pietro Bembo's "Gli Asolani", «Modern language Notes», LXXXVII (1972), I, pp. I34-39; C. Fahy, A note on the printing of the Isos aldine edition of P. Bembo's "Asolani", "The Library», XXVII (1972), pp. I $36-42$; e la successiva ricostruzione di Dilemmi nelle pagine introduttive all'edizione critica del dialogo. La forma originaria della missiva, leggermente diversa da quella andata a stampa, si legge in Pietro Bembo, Lettere, edizione critica a c. di E. Travi, I, Bologna, Commissione per i testi in lingua, 1987 (Collezione di opere inedite o rare, I4I), i92, pp. 177-78, e in Piettro Bembo - Lucrezia Borgia, La grande fiamma, a c. di G. Raboni, Milano, Archinto, 1989, 23, pp. $51-52$.

3. Basti, oltre all'introduzione di Dilemmi, il rinvio ai seguenti contributi: C. Clough, Pietro Bembo, Madonna G., Berenice and Veronica Gambara, «Commentari dell'Ateneo di Brescia per l'anno 1963", A.A. CLXII, Brescia, Fratelli Geroldi, 1965, pp. 209-27; L. Fortini, Itinerari di scrittura: Pietro Bembo e "Gli Asolani", "La Rassegna della Letteratura Italiana», LXXXVIII (1 984), pp. 389-98; G. Dilemmi, «Andrem di pari): Maria Savorgnan e "Gli Asolani" del Bembo, "Quaderni del Dipartimento di Lingue e Letterature neolatine», IV (1988-1989), pp. 49-72. Utile anche E. Scarpa, Qualche proposta (e qualche ipotesi) per i primi "Asolani”, «Studi di Filologia Italiana», LII (1994), pp. 93-109. Interessanti cenni al tessuto autobiografico in A. Acciani, "Quasi in uno speccbio riguardando»: l'io e l'altro negli "Asolani" del Bembo, in Scritture di sé. Autobiografismi e autobiografie, a c. di F. Pappalardo, Napoli, Liguori, 1994, pp. 21-56.

4. L'intera corrispondenza è raccolta in: Maria Savorgnan - Pietro Bembo, Carteggio d'amore (I500-I50I), a c. di C. Dionisotti, Firenze, Le Monnier, 1950, da cui si cita. Per le lettere di Bembo si segnala anche la numerazione secondo l'edizione Travi.

5. Si rinvia specialmente a G. Gorni, Veronica e le altre: emblemi e cifre onomastiche nelle rime del Bembo, in Veronica Gambara e la poesia del suo tempo nell'Italia settentrionale. Atti del Convegno (Brescia - Correggio, 17-19 ottobre 1985), a c. di C. Bozzetti, P. Gibellini, E. Sandal, Firenze, Olschki, 1989, pp. 37-57.

6. Savorgnan - Bembo, Carteggio d'amore, 74, pp. i 29-30 (Bembo, Lettere, I, i 29, pp. i 22-23).

7. Dionisotti ipotizza anzi che la datazione tràdita sia eccessivamente bassa, implicitamente suggerendo uno slittamento indietro di qualche giorno: Savorgnan - Bembo, Carteggio d'amore, p. I69.

8. Si veda la lettera 70 di Maria, SAvorgnan - Bembo, Carteggio d'amore, pp. 38-40 (a p. 38 la citazione).

9. Questa la prima quartina: «Hor ch'è estinta la fiama e sciolto il nodo / e la prigion aperta di martiri, / riposerete o mei stanchi suspiri, / né più rinforzerete il focho al chiodo». Savorgnan - Bembo, Carteggio d'amore, 77, p. 43.

го. Bемво, Gli Asolani, II xxхıг, p. 3 го.

I I. Bемво, Gli Asolani, I xix, p. 238. 
I 2. вемво, Gli Asolani, III I, p. 3 I 4 .

I 3. Savorgnan - Bembo, Carteggio d'amore, i i, p. 55 (Bembo, Lettere, I, 57, p. 48).

I4. Savorgnan - Bembo, Carteggio d'amore, 34, p. i9. Che la Savorgnan fosse in possesso di una spiccata sensibilità ritmica è confermato dalla frequenza con cui, nelle lettere da lei inviate a Pietro, si inseriscono clausole endecasillabiche già rilevate da Dionisotti. La familiarità con i Trionfi e l'uso che Bembo ne fa, mentre confermano la ricercata letterarietà della corrispondenza con Maria, suggeriscono un'ipotetica pista interpretativa, indicando proprio nell'amara apoteosi dell'amore descritta da Petrarca una possibile fonte del primo libro asolano.

I 5. Savorgnan - Bembo, Carteggio d'amore, 47, p. 87 (Bembo, Lettere, I, 96, p. 83 ).

I6. Sull'edizione I 548 delle Rime di Pietro Bembo si rinvia, in particolare, a: M. Pecoraro, Rassegna bembiana, «Lettere Italiane», XV (I 963), 4, pp. 446-84; P. Sabbatino, Sulla tradizione a stampa delle "Rime" del Bembo, "Studi e problemi di critica testuale», XXVIII (1984), pp. 57-98; P. Trovato, Per la storia delle "Rime" del Bembo, «Rivista di Letteratura Italiana», IX (I99I), 3, pp. 465-508.

I 7. Lo strambotto Città con più sudor posta e cresciuta è il numero xil delle Rime rifiutate (PIETRO Bembo, Prose e rime, a c. di C. Dionisotti, Torino, UTET, i 960, p. 684). L'altro strambotto (primo in ordine di tempo) fu inviato a Maria con lettera ascrivibile al luglio i 500 e inizia così: Chi rompe ne l'Egeo, se poi vi riede (Вемвo, Prose e rime, x, pp. 682-83). Il terzo, non inserito nella silloge delle rime ricusate, si legge in Pietro Bembo, Gli Asolani e le Rime, a c. di C. Dionisotti, Torino, UTET, I932, p. 284. Metricamente omogeneo allo schema dello strambotto è anche un componimento inserito nella ne varietur, Qual meraviglia, se repente sorse, che Dionisotti preferisce però interpretare come stanza (B Емвo, Prose e rime, Lxv, p. 562). Sulle fortune del genere strambottistico tra Quattro e Cinquecento, oltre all'utilissima mappatura raccolta in G. La Face Bianconi - A. Rossi, Sulla diffusione del repertorio strambottistico di fine Quattro- inizio Cinquecento: premesse e bibliografia, «Schifanoia», X (1 990), pp. I 29-60, si possono consultare: P. Vecchi Galli, Strambotti anonimi quattrocenteschi da un codice della Colombina di Siviglia, in Studi in onore di Raffaele Spongano, Bologna, Boni, I980, pp. 173-93; D. Delcorno Branca, Dal Poliziano a Serafino, in Umanesimo e Rinascimento a Firenze e Venezia. Miscellanea di studi in onore di Vittore Branca, Firenze, Olschki, I983, II-2, pp. 423-50; A. Rossi, Lirica volgare del primo Cinquecento: alcune annotazioni, in Forme e vicende per Giovanni Pozzi, a c. di O. Besomi, G. Gianella, A. Martini, G. Pedrojetta, Padova, Antenore, I 988 (Medioevo e Umanesimo, 72), pp. I 23-57; Id., "Prima ch'i" die principio a' miei strambotti», in Di selva in selva. Studi offerti a Pio Fontana, a c. di P. Di Stefano e G. Fontana, Bellinzona, Edizioni Casagrande, I993, pp. 25 I-64; D. Delcorno Branca, Per il linguaggio dei rispetti del Poliziano, «Rinascimento», s. II, XXXV (I995), pp. 3 I-66.

i 8. Matteo Maria Boiardo, Amorum libri tres, a c. di T. Zanato, Torino, Einaudi, i 998 (Nuova raccolta di classici italiani annotati, I6), I xxxvin, pp. I I 3-I 5. L'identico motivo torna in Orlando Innamorato III I I-2.

I 9. L'idea è affacciata da Gorni nel contributo Veronica e le altre cit., pp. 48-49. Ripresa da Dilemmi nell'intervento citato, pubblicato nei «Quaderni del Dipartimento di Lingue e Letterature neolatine» («Andrem di pari»: Maria Savorgnan e "Gli Asolani" del Bembo), e nell'introduzione agli Asolani, è confermata dallo stesso Gorni nel volume di Poeti del Cinquecento, I: Poeti lirici, burleschi, satirici e didascalici, a c. di G. Gorni, M. Danzi, S. Longhi, Milano-Napoli, Ricciardi, 200 I (La Letteratura Italiana - Storia e testi, 23/I), p. I 32 n.

20. Sul motto, assunto a slogan dell'amore con la Savorgnan, oltre al citato saggio di Gorni, si veda già Dionisotti, nell'introduzione e nelle note a SAvorgnan - BEmbo, Carteggio 
d'amore. Qualche spunto anche in M. Pozzi, «Andrem di pari all'amorosa face». Appunti sulle lettere di Maria Savorgnan, in Les femmes écrivains en Italie au Moyen Âge et à la Renaissance. Acte du colloque international (Aix-en-Provence, I 2-I4 novembre 1992), Aix-en-Provence, Publications de l’Université de Provence, I994, pp. 87-Ior.

21. «Da alquanti giorni in qua io posso dire verissimamente, che tutto il mio vivere, o lieto o tristo, ha nel vostro o lietamente o altramente vivere la radice». SAvorgnan - Bembo, Carteggio d'amore, 35, p. 7 I (Bемвo, Lettere, I, 84, p. 68).

22. Savorgnan - Bembo, Carteggio d'amore, 40, p. 78 (Bembo, Lettere, I, 89, p. 74).

23. Savorgnan - Bembo, Carteggio d'amore, 48, p. 90 (Bembo, Lettere, I, 97, p. 84).

24. Savorgnan - Bembo, Carteggio d'amore, 4I, p. 79 (Bembo, Lettere, I, 90, p. 75). Il corsivo è citazione petrarchesca, $R v f .72,36$.

25. Sono i componimenti xv, Lv, LXxiI e LxxiII delle Rime, cui si aggiunge la canzone Se ne la prima voglia mi rinvesca, contenuta negli Asolani, III Ix. Gorni, Veronica e le altre cit., pp. 48-49.

26. Bемво, Prose e rime, LxxiII, v. I I, p. 568. Sul trattamento riservato ai brani in versi, nel transito dalla princeps all'edizione i 530 degli Asolani $\mathrm{cfr}$. G. Dilemmi, La parte delle rime nei secondi "Asolani" (I530), in Studi di letteratura italiana offerti a Dante Isella, Bibliopolis, Napoli, I 983 , pp. 8 I-IO2.

27. Gorni, Veronica e le altre cit., p. 45.

28. Si tratta dei sonetti cxuvini, CLi e CLII, per i quali si veda Gorni, Veronica e le altre cit., p. 5 I n.

29. Felice è parola rima in xv (v. I, nella forma derivata infelice) e LV (v. I s). È inoltre presente, fuor di rima, ancora in XV (V. I), in LXXII (v. 9 - dove radice non è in clausola), e nella canzone Se ne la prima voglia mi rinvesca (v. 22).

30. Sul significato e sull'origine dei simboli mariani accennati: L. Bartoli, Lessico di simbologia mariana, Padova, Libreria Editrice Gregoriana, 1988, ad voces (dove si segnala anche il gioco paronomastico tra il nome della Vergine, il sostantivo mare e l'aggettivo amaro. Sembra dimostrabile che un identico sistema concettuale, desunto o meno dal modello, valga anche per le rime dedicate alla Savorgnan, dove frequente è il ricorso all'idea di amarezza, non a caso presente anche nello strambotto in analisi). Sui loci scritturali che stanno alla base del sistema simbolico: Encbiridion marianum biblicum patristicum, a c. di D. Casagrande, Roma, Sumptibus "Cor Unum" Figlie della Chiesa, I974. Un ricco repertorio di testi e fonti si legge in Maria. Testi teologici e spirituali dal I al XX secolo, a c. della Comunità di Bose, con un saggio introduttivo di E. Bianchi, Milano, Mondadori, 2000 (I Meridiani - Classici dello Spirito). Sulla diffusione del culto mariano in età rinascimentale è di qualche utilità: Arte, religione, comunità nell'Italia rinascimentale e barocca. Atti del convegno di studi in occasione del V centenario di fondazione del Santuario della Beata Vergine dei Miracoli di Saronno (I498-1998), a c. di L. Saccardo e D. Zardin, Milano, Vita e Pensiero, 2000 (in particolare G. Cracco, Culto mariano e istituzioni di Chiesa tra medioevo ed età moderna, pp. 25-52, e T. Verdon, 'Domus aurea': santuari mariani nel '400 e nel' 'soo, pp. 83-93).

31. Savorgnan - Bembo, Carteggio d'amore, i 2, p. 56 (Bembo, Lettere, I, 58, p. 49). Altre occorrenze del motivo si leggono nei passi seguenti, dei quali i primi due scritti da Maria, $\mathrm{i}$ successivi di mano di Pietro: «Le rose mi sono gratissime, a l'incontro di le qual non ho che mandarvi, salvo un poco de basilicho et una certa erba che porta il nome di quel che questi 


\section{Due rime giovanili del Bembo}

giorni $\mathrm{mi}$ ha fato mandarvi tinte molte carte, e come quel da me non si parte mai» (Savorgnan - Bembo, Carteggio d'amore, 7, p. 6); «La stagion di la rosa è ben venuta [...]. Per altro vorei che circhasti o disiati vedermi che per la rosa» (SAvorgnan - Bembo, Carteggio d'amore, 56, p. 33); «Il segno, che io porto di voi nella mia persona, è dentro in tutto 'l cuore, voi tutta viva e movente, e ora dolce e quando amara, sì come solete meco essere, quando io vi sono innanzi. Di fuora, è una dolce macchia di quel colore, di cui sogliono essere le porporine rose, grande quanto picciol rosa, rimastami la felice sera delle mille cose» (Savorgnan - Bembo, Carteggio d'amore, 54, p. io i [Bembo, Lettere, I, io6, p. 98]); «La vostra vermiglia rosa, che sapete, la quale avea già perduto ogni suo vigore, poi che ella dal bello avorio delle vostre mani fu tocca, ha ripreso colore e vita, et essi fatta più fresca d'assai che ella prima non era: dolcissimo miracolo d'Amore, se non che appo voi nessuna cosa può essere miracolo altra che voi, che sete dolcissimo miracolo, e d'Amore e della Natura» (Savorgnan - Bembo, Carteggio d'amore, 58, p. ios [Bembo, Lettere, I, i i I, p. IO2]).

32. Вемво, Lettere, I, i 8 I, p. I69.

33. La lettera, I 48 dell'epistolario curato da Travi, è tràdita da codici e stampe con data Io febbraio I 503 . Morsolin, sulla scorta di alcuni rilievi biografici, la posticipa di due anni e la interpreta senz'altro come destinata a Lucrezia, cfr. B. Morsolin, Pietro Bembo e Lucrezia Borgia, «Nuova Antologia», XX (1885), I 5, pp. 388-422, pp. 417-1 8. Lo segue Giulia Raboni nell'edizione dell'epistolario (si veda la nota della curatrice in BEMBo - Borgia, La grande fiamma, pp. 98-99). Quale che sia la cronologia esatta, sembra accettabile l'ipotesi che il nome di Madonna Nicola servisse a Bembo come schermo per comunicare liberamente con la duchessa. Per il carteggio con Lucrezia si cita da Bembo - Borgia, La grande fiamma, segnalando tra parentesi il rimando all'edizione Travi.

34. Вемво - Borgia, La grande fiamma, 27, p. 58 (Bемво, Lettere, I, i 48, p. I40).

35. Si leggano, a confronto, la lettera 27 di Bembo e Gli Asolani, II xxirı.

36. Bемвo - Borgia, La grande fiamma, 2, p. 27 (Bемвo, Lettere, I, I 53 , p. I 46). Sull'episodio si veda la ricostruzione di M. Bellonci, Lucrezia Borgia. La sua vita e $i$ suoi tempi, Milano, Mondadori, I940, p. 402.

37. Sulle rime di Pietro ascrivibili a Lucrezia Borgia e sugli emblemi dell'amore tra i due: Gorni, Veronica e le altre cit., pp. 54-55.

38. Bembo - Borgia, La grande fiamma, 3, p. 28 (Bembo, Lettere, I, I 5 5, p. I48). Il sonetto registra un discreto numero di varianti nel manoscritto Marc. It. IX. I43, prima raccolta organica delle rime del Bembo, mai andata alle stampe e allestita, circa il i 5 I o- I 5 I I, per la duchessa di Urbino Elisabetta Gonzaga. In particolare: v. 2, campo è sostituito da regno; v. 4, si inverte l'ordine tra scoprir e sol; v. 7, Dell'interno mio mal evolve in Del stato interno mio; v. 9, crederei diviene sperarei, cfr. C. Vela, Il primo canzoniere del Bembo (ms. Marc. It. IX. I43), «Studi di Filologia Italiana», XLVI (i 988), pp. I63-25 I (il sonetto è a p. 227). L'explicit, intatto nel Marciano, risulta modificato nell'edizione i 530 dove, pur conservando i ritocchi ai vv. 2 e 9 , Bembo ripristinò parzialmente la forma originaria, intervenendo tuttavia sul verso finale: «La medicina è poca, il languir molto» (è questa la redazione accolta da Gorni nei Poeti del Cinquecento cit., p. 59). Sistemando le rime per la ne varietur, Bembo recuperò il testo spedito alla Borgia, mantenendo unicamente le varianti del verso conclusivo.

39. Alcune, suggestive ipotesi si leggono in Bellonci, Lucrezia Borgia cit., p. 403.

40. Come segnalato da Gorni (Poeti del Cinquecento cit., p. 59), l'immagine del cristallo compare già, con l'identica funzione, nella corrispondenza con Maria: «Sanno gl'Idii con che animo io vi parlo, e volessero essi che voi poteste vedere il cuor mio. Ma voi ad ogni 
modo il vederete più chiaramente che se io fossi un cristallo, e caro vi fia per aventura lo averlo vedutom, Savorgnan - Bembo, Carteggio d'amore, 48, p. 90 (Bembo, Lettere, I, 97 , p. 85$)$.

4I. Bемво, Gli Asolani, II xxviII, vv. 49-54, p. 300. L'immagine doveva esercitare uno speciale fascino su Bembo, che se ne avvalse anche, in apertura del dialogo (I I), per descrivere la specificità e le funzioni della scrittura, quasi specchio che, riflettendo le cose passate, si offre all'uomo come strumento di educazione. Si veda, per questo motivo: C. De Bellis, Il giardino dei ragionamenti. Note sugli "Asolani" di Bembo, in Letteratura italiana e arti figurative. Atti del XII Convegno dell'Associazione Internazionale per gli Studi di Lingua e Letteratura Italiana (Toronto - Hamilton - Montreal, 6-1o maggio I98 5), a c. di A. Franceschetti, Firenze, Olschki, 1988 (Biblioteca dell'«Archivum Romanicum», 208), pp. 463-72.

42. Nei Rerum Vulgarium Fragmenta si registrano undici occorrenze del verbo languire (anche in funzione sostantivata), contro cinque occorrenze di strazio - straziare.

43. Gorni, Veronica e le altre cit., p. 54.

44. Dionisotti, nota a Bembo, Prose e rime, p. 54I. Per il brano della lettera a Lucrezia si veda Bembo - Borgia, La grande fiamma, 32, p. 66 (Bembo, Lettere, I, 247, p. 24I). Con il motto, tratto da Petrarca $(R v f$. I०8, I I), si chiude il secondo dei due sonetti promessi alla duchessa di Ferrara.

45. Si vedano, tra le lettere indirizzate alla Borgia, la n. is (174 dell'edizione Travi), dove Bembo ripropone l'idea per cui l'amata è l'altra metà dell'innamorato (concetto anticipato negli Asolani II xI-XII e replicato nelle Stanz̨, ottava 47, quattro anni più tardi); e la n. 20 ( 187 dell'edizione Travi, dove tuttavia risulta indirizzata a Madonna $\mathrm{L}<$ isabetta $>$ da $\mathrm{S}<$ iena $>$ ) che allude al triplice percorso di fruizione dell'amore (vista, udito e pensiero), già difeso da Gismondo nel II libro del dialogo (Gli Asolani II xxII-XxviII).

46. È il caso, a titolo d'esempio, dei citati sonetti xLII-XLIII, costruiti sull'impresa petrarchesca; ovvero dei tre sonetti lucreziani dedicati al sogno, LxxxvinI-xc delle Rime. 